\section{AB0091 INHIBITION OF AUTOPHAGY PREVENTS PROGRESSION OF FIBROSIS IN MURINE MODELS OF SYSTEMIC SCLEROSIS}

A. Zehender ${ }^{1}$, Y. N. Li ${ }^{1}$, N. Y. Lin ${ }^{1}$, A. H. Györfi ${ }^{1}$, A. Soare ${ }^{1}$, C. Bergmann ${ }^{1}$, A. Ramming ${ }^{1}$, G. Schett ${ }^{1}$, J. H. W. Distler ${ }^{1} .{ }^{1}$ Medicine 3 of the University Clinic Erlangen, Medizin 3, Erlangen, Germany

Background: Autophagy is catabolic process allowing cells to degrade unnecessary or dysfunctional cellular organelles. Failure of appropriate regulation of autophagy, however, can severely perturb tissue homeostasis. Recent studies demonstrate that autophagy is activated in several fibrotic diseases such as liver fibrosis, renal interstitial fibrosis, cardiac fibrosis.

Objectives: The objective of this work was to characterize the activation of autophagy in systemic sclerosis (SSc) and to decipher its role in the pathogenesis of SSc Methods: Activation of autophagy in skin samples of patients and murine models of SSc was assessed by co-staining of LC3B and P62 with the lysosomal marker LAMP2. The role of the autophagy was investigated in the model of bleomycin-induced dermal fibrosis. Beclin1 was overexpressed using adenovirus encoding for Beclin1. To knockdown Atg7 in vivo was achieved by subcutaneous injections of Atg7 siRNA or non-targeting siRNA. In vivo, 3-methyladenine (3-MA) was administered i.p. in a concentration of $15 \mathrm{mg} / \mathrm{kg}$ ones daily. Protein expression was measured by Western blot. Target genes were analyzed by qPCR. To monitor the autophagic flux, we generated adenoviral vectors encoding for tandem fluorescent-tagged LC3 (mRFP-EGFP-LC3).

Results: In the present study, we demonstrate that autophagy is activated in fibroblasts in SSc skin and also in experimental fibrosis models as compared to respective non-fibrotic control tissue with enhanced activity in in vivo and in vitro autophagy reporter studies. The aberrant activation of autophagy had profound stimulatory effects on fibroblasts. Activation of autophagy by forced expression of BECLIN1 promoted fibroblast-to-myofibroblast transition and stimulated the collagen release by cultured human fibroblasts and induced fibrosis in murine model. Nevertheless, inhibition of autophagy can deactivate myofibroblasts and induce regression of tissue fibrosis. Knockdown of ATG7 or BECLIN1 in human fibroblasts reduced the expression of aSMA and the number of stress fibers in myofibroblasts, indicating re-differentiation of myofibroblasts into resting fibroblasts upon inhibition of autophagy. Similar results were obtained with the autophagy inhibitors CQ and 3-MA. In vivo, siRNA mediated knockdown of Atg7 effectively prevented progression of fibrosis in a model of established bleomycin-induced skin fibrosis. Inactivation of autophagy decreased dermal thickness, myofibroblast counts and hydroxyproline content to below pretreatment levels, indicating regression of bleomycin-induced skin fibrosis. In addition, treatment of mice with the autophagy inhibitor 3-MA ameliorated bleomycin-induced skin fibrosis.

Conclusion: We demonstrate that autophagy activity is enhanced in fibroblasts of SSc patients and in murine models of SSc. The increased activation of autophagy induces fibroblast-to-myofibroblast transition and promotes fibrotic tissue remodeling. However, inhibition of autophagy can deactivate myofibroblasts and induce regression of tissue fibrosis.

REFERENCES:

[1] Wynn, T. Cellular and molecular mechanisms of fibrosis. J Pathol 214, 199210 (2008).

[2] Klionsky DJ, Abeliovich $\mathrm{H}$, Agostinis P, et al. Guidelines for the use and interpretation of assays for monitoring autophagy in higher eukaryotes. Autophagy 4, 151-175 (2008).

[3] Wang, CW \& Klionsky, DJ. The molecular mechanism of autophagy. Mol Med 9, 65-76 (2003).

[4] Hernández-Gea V, Ghiassi-Nejad Z, Rozenfeld R, et al. Autophagy releases lipid that promotes fibrogenesis by activated hepatic stellate cells in mice and in human tissues. Gastroenterology 142, 938-946 (2012)

Disclosure of Interests: Ariella Zehender: None declared, Yi-Nan Li: None declared, Neng-Yu Lin: None declared, Andrea-Hermina Györfi: None declared, Alina Soare: None declared, Christina Bergmann: None declared, Andreas Ramming: None declared, Georg Schett: None declared, Jörg H.W. Distler Consultant of: Actelion, Active Biotech, Anamar, ARXX, Bayer Pharma, Boehringer Ingelheim, Celgene, Galapagos, GSK, Inventiva, JB Therapeutics, Medac, Pfizer, RuiYi and UCB., Grant/research support from: Anamar, Active Biotech, Array Biopharma, aTyr, BMS, Bayer Pharma, Boehringer Ingel-heim, Celgene, Galapagos, GSK, Inventiva, Novartis, Sanofi-Aventis, RedX, UCB., Employee of: stock owner of 4D Science and Scientific head of FibroCure

DOI: 10.1136/annrheumdis-2021-eular.819

\section{AB0092 \\ ANTISYNTHETASE SYNDROME: CLINICAL PROFILE, SEROLOGIC AND TREATMENTS USED IN A COHORT OF PATIENTS FOLLOWED AT THE VIRGEN MACARENA HOSPITAL}

P. Muñoz Reinoso ${ }^{1}$, I. García Hernández ${ }^{1}$, M. Ferrer Galván², F. J. Toyos Sáenz de Miera ${ }^{1}$, L. Fernández de la Fuente Bursón ${ }^{1}$, J. J. Pérez Venegas ${ }^{1} .{ }^{1}$ Hospital
Universitario Virgen Macarena, Reumatología, Sevilla, Spain; ${ }^{2}$ Hospital Universitario Virgen Macarena, Neumología, Sevilla, Spain

Background: The antisynthetase syndrome (SAS) is characterized by the presence of antisynthetase antibodies, anti-JO1, PL7 y PL12 are the most common and the classic triad of myositis, arthritis, and diffuse interstitial lung disease (ILD) ${ }^{1}$. Most patients present incomplete forms and the severity of the ILD determines the prognosis of the disease ${ }^{2}$.

Objectives: to analyze epidemiological, clinical and serological characteristics and treatments used in a cohort of patients with SAS.

Methods: descriptive study of review of medical records. Data were collected from 15 patients with SAS followed in the Rheumatology and Pneumology consultations of the Virgen Macarena Hospital (Seville) in the last 10 years. The analysis was carried out using the $\mathrm{R}$ software.

Results: 15 patients were included, 8 men and 7 women. The median age was 56 years (33-77). Seven patients (47\%) used to smoke. Four patients $(27 \%)$ me the classical triad. All of them presented ILD and 8 patients $(53 \%)$ had arthritis and / or myositis. Five (33\%) had mechanic's hands and six of them (40\%) presented Raynaud. Seven (47\%) suffered from dyspnea before the SAS diagnosis. The median diagnostic delay was 1 month (0-43). Seven (47\%) patients had anti-JO1, 1 (7\%) anti-PL7, 2 (13\%) anti-PL12 and 2 (13\%) patients anti-Ro52. Radiological patterns detected by HRCT were: 5 (33\%) NINE, 4 (37\%) NIU and $6(40 \%)$ others. The initial treatment included mostly $(66 \%)$ glucocorticoids (GC) and one or more cFAME. In maintenance, mycophenolate was used in 7 patients $(47 \%)$, cyclosporine $5(33 \%)$, cyclophosphamide in 3 cases $(20 \%)$, azathioprine in 3 patients $(20 \%)$ and methotrexate in 3 of them $(20 \%)$. Four $(37 \%)$ patients required a combination of DMARDs and 2 cases needed (13\%) biological therapy, Rituximab and Tocilizumab. Changes in the mean value of the initial respiratory function tests (FVC1 and DLCO1) and during follow-up (FVC2 and DLCO2) were not relevant (FVC1 81.5\% [42-110], FVC2 81\% [59-115]; DLCO1 $83 \%$ [10-112], DLCO2 $80.5 \%$ [47-108]). Nine patients $(60 \%)$ remained clinically stable and 3 patients $(20 \%)$ progressed radiologically. Four patients died from ILD progression.

Conclusion: In this study, the incomplete diagnosis of SAS predominated. The most detected antibody was anti-JO1. ILD is present in all cases, with NINE being the most frequent pattern so multidisciplinary management is necessary. Most used treatments were GC and FAMES combined, some cases required biological therapy.

REFERENCES:

[1] Irazoque F, et al. Epidemiology, etiology and classification. Reumatol Clin 2009;5:2-5.

[2] Johnson C, et al. Clinical and pathologic differences in interstitial lung disease based on antisynthetase antibody type. Respir Med. 2014; 108(10):1542-8. Disclosure of Interests: None declared DOI: 10.1136/annrheumdis-2021-eular.1427

\section{AB0093 CLINICAL AND SEROLOGICAL CHARACTERISTICS OF SYSTEMIC SCLEROSIS: EXPERIENCE FROM A TERTIARY CARE CENTER IN PAKISTAN}

S. Asif ${ }^{1,2,2}$, M. Haroon ${ }^{1}$, A. Khan2, M. Faiq ${ }^{1}{ }^{1}$ Fatima Memorial Hospital, Lahore, Rheumatology, Lahore, Pakistan; ${ }^{1}$ Fatima Memorial Hospital, Lahore, Rheumatology, Lahore, Pakistan

Background: Systemic sclerosis (SS) is less studied Connective tissue disease in our population. It is characterized by different manifestations which if left undiagnosed and untreated lead to serious complications. The hallmark of this disease is fibrosis of various organs including skin and also involving pulmonary, gastrointestinal and cardiovascular system. We aim to evaluate the clinical and serological characteristics of SS in our population. In addition, we evaluated the prevalence of Fibromyalgia and Depression in patients with SS.

Objectives: We aimed to determine Clinical and Serological Characteristics of Systemic Sclerosis visiting our department of Rheumatology, Fatima Memorial hospital, Lahore.

Methods: It is a cross-sectional study of 38 patients visiting outdoor and indoor in Rheumatology department of Fatima Memorial Hospital from September 2019 to January 2020. All systemic sclerosis patients with age 16 years or above were included in this study. After taking informed consent, we filled the performas to record all the required information. We evaluated the clinical, serological and radiological features of Systemic Sclerosis.

Results: A total of 38 patients were included in the study with mean age was $34.47 \pm 1.53$ years. Female gender comprised of $32(84.2 \%)$, with male to female ratio of $1: 5.3$. Thirty-four (89.5\%) had developed Raynaud phenomenon and sclerodactyly was found in 34(89.5\%), digital ulcers in $25(65.8 \%)$, and tendon friction rub in $12(31.6 \%)$ patients. Skin tightness proximal to elbow was present in $9(23.7 \%)$. Microstomia (decreased mouth opening) was present in $32(84.2 \%)$ patients. Twenty-eight (73.7\%) patients had arthritis. Respiratory symptoms comprised of shortness of breath in 36(94.7\%), palpitation in $24(63.2 \%)$ and chest 
pain in $7(18.4 \%)$. Gastrointestinal (Gl) symptoms comprised of regurgitation in $31(81.5 \%)$ and dysphagia in $14(36.8 \%)$. Interstitial lung disease was present in $30(78.9 \%)$ patients, with higher prevalence in diffuse scleroderma $(100 \%)$ than in limited scleroderma $(70 \%)(p=0.01)$. Pulmonary hypertension was present in 18 patients, with significantly higher prevalence in diffuse disease $(57.1 \%)$, that was secondary to interstitial lung disease and in limited disease it was found in $(11.8 \%)(p<0.01)$. Thirty $(78.9 \%)$ patients were found to have restricted disease on pulmonary function tests.

Obstetric history showed a higher prevalence of primary infertility in at least 6 $(15.8 \%)$ patients, with significantly higher prevalence in limited systemic sclerosis disease as compared to diffuse disease $(23.5 \%$ vs $9.5 \%$, and $p=0.05)$. Fibromyalgia diagnosed as per ACR criteria was present in 7 (18.4\%) patients, and depression assessed by Hospital Anxiety and depression (HADS) score was present in $10(26.3 \%)$ patients.

Anti nucleic acid antibody (ANA) was found positive in $30(78.9 \%)$ patients. Anti Scl-70 antibodies were in $24(63.2 \%)$ patients, with significant association with diffuse disease ( $85 \%$ vs $35.3 \%$ and $p<0.01$ ), while anti centromere antibodies were present in 20 (52.6\%) patients; significantly higher in limited disease $(94.2 \%$ vs $19.0 \%$, and $p<0.01)$.

Conclusion: Scleroderma is a very important, autoimmune multisystem disease. It has female preponderence. Raynaud phenomenon is the most initial clinical feature followed by other manifestations of variable course and disease severity. Interstitial lung disease and pulmonary hypertension were the most important complication found in our patients which has poor prognosis. So, It is imperative to early diagnose and treat the disease manifesations to prevent future complications.

Keywords: Scleroderma, Systemic sclerosis.

DISCLOSURE OF INTEREST:

Sadia Asif: None declared, Muhammad Haroon: None declared, Dr Asadullah Khan: None declared, Dr. Muhammad Faiq: None declareDOI: 10.1136/ annrheumdis-2021-eular.1635

\section{AB0094 INCREASE OF ENDOTHELIAL PROGENITOR CELLS IN SYSTEMIC SCLEROSIS-ASSOCIATED INTERSTITIAL LUNG DISEASE}

V. Pulito-Cueto $^{1}$, S. Remuzgo Martinez ${ }^{1}$, F. Genre ${ }^{1}$, B. Atienza-Mateo ${ }^{1,2,3}$, V. M. Mora-Cuesta ${ }^{1,4}$, D. Iturbe-Fernández ${ }^{1,4}$, L. Lera-Gómez ${ }^{1}$, R. PérezFernández ${ }^{1}$, D. Prieto-Peña ${ }^{1,3}$, V. Portilla ${ }^{1,3}$, R. Blanco ${ }^{1,3}$, A. Corrales ${ }^{1,3}$, J. M. Cifrián-Martínez ${ }^{1,4,5}$, R. López-Mejías ${ }^{1}$, M. A. González-Gay ${ }^{1,3,5,6}{ }^{1}{ }^{1}$ IDIVAL, Research Group on Genetic Epidemiology and Atherosclerosis in Systemic Diseases and in Metabolic Bone Diseases of the Musculoskeletal System, Santander, Spain; ${ }^{2}$ Hospital Universitario Marqués de Valdecilla, 'López Albo Post-Residency Programme, Santander, Spain; ${ }^{3}$ Hospital Universitario Marqués de Valdecilla, Department of Rheumatology, Santander, Spain; ${ }^{4}$ Hospital Universitario Marqués de Valdecilla, Department of Pneumology, Santander, Spain; ${ }^{5}$ Universidad de Cantabria, School of Medicine, Santander, Spain; ${ }^{6}$ Faculty of Health Sciences, University of the Witwatersrand, Cardiovascular Pathophysiology and Genomics Research Unit, School of Physiology, Johannesburg, South Africa

Background: Endothelial progenitor cells (EPC), involved in vasculogenesis and endothelial tissue repair, have been described as relevant players in vascular and connective tissue diseases [1-2]. In this regard, a previous study of our group disclosed that the degree of EPC frequency may help to identify the presence of interstitial lung disease (ILD) in rheumatoid arthritis patients [3]. Given that ILD is the main cause of mortality in patients with systemic sclerosis (SSc) [1, 4-6], the understanding of the role of EPC in the mechanism of SSc-ILD+ vasculopathy is crucial.

Objectives: To assess the potential role of EPC on vascular dysfunction associated with the presence of ILD in patients with SSc.

Methods: Peripheral venous blood was collected from a total of 39 patients with SSc, 20 with ILD (SSc-ILD ${ }^{+}$) and 19 without ILD (SSc-ILD'). All subjects were recruited from the Rheumatology and Pneumology departments of Hospital Universitario Marqués de Valdecilla, Santander, Spain. Quantification of EPC was analyzed by flow cytometry. EPC were considered as CD $34^{+}, \mathrm{CD}^{\mathrm{LOw}}{ }^{\mathrm{Low}}, \mathrm{CD} 09^{+}$ and $\mathrm{CD} 133^{+}$

Results: Statistically significant differences in EPC frequency between patients with SSc-ILD ${ }^{+}$and patients with SSc-ILD were disclosed. Specifically, an increase of EPC frequency was observed in SSc-ILD ${ }^{+}$patients when compared to patients with SSc-ILD' (mean \pm standard deviation: $0.033 \pm 0.012$ versus 0.021 \pm 0.017 , respectively, $p=0.012$ ).

Conclusion: Our results suggest a potential role of EPC on vascular damage associated with the manifestation of ILD in patients with SSc.

REFERENCES:

[1] Eur J Rheumatol 2020;7(Suppl 3):S139-S146.

[2] Arthritis Rheum 2009;60(11):3168-79.

[3] J Clin Med 2020;9(12):4098.
[4] Ann Rheum Dis 2007;66(7):940-4.

[5] Rheumatology (Oxford) 2010;49(12):2375-80.

[6] Eur Respir Rev 2015;24(135):102-14.

Acknowledgements: Personal funds, VP-C: PREVAL18/01 (IDIVAL); SR-M: RD16/0012/0009 (ISCIII-ERDF); LL-G: INNVAL20/06 (IDIVAL); RP-F: START PROJECT (FOREUM); RL-M: Miguel Servet type I CP16/00033 (ISCIII-ESF).

Disclosure of Interests: Verónica Pulito-Cueto: None declared, Sara Remuzgo Martinez: None declared, Fernanda Genre: None declared, Belén Atienza-Mateo: None declared, Victor Manuel Mora-Cuesta: None declared, David Iturbe-Fernández: None declared, Leticia Lera-Gómez: None declared, Raque Pérez-Fernández: None declared, Diana Prieto-Peña: None declared, Virginia Portilla: None declared, Ricardo Blanco Speakers bureau: Abbvie, Pfizer, Roche Bristol-Myers, Janssen and MSD, Consultant of: Abbvie, Pfizer, Roche, Bristol-Myers, Janssen and MSD, Grant/research support from: Abbvie, MSD and Roche, Alfonso Corrales: None declared, Jose Manuel Cifrián-Martínez: None declared, Raquel López-Mejías: None declared, Miguel A González-Gay Speakers bureau: Pfizer, Abbvie, MSD, Grant/research support from: Pfizer, Abbvie, MSD

DOI: 10.1136/annrheumdis-2021-eular.2350

\section{$\mathrm{AB} 0095$}

EXPRESSION AND PATHOGENIC ROLES OF INTEGRIN FAMILY GENE IN SYSTEMIC SCLEROSIS

D. $\mathrm{Xu}^{1}, \mathrm{~T} . \mathrm{Li}^{1}, \mathrm{R} . \mathrm{Mu}^{1} .{ }^{1}$ Peking University Third Hopsital, Department of Rheumatology and Immunology, Beijing, China

Background: Emerging evidence have shown that some integrin members are associated with inflammation and fibrosis in systemic sclerosis (SSc) patients ${ }^{[1-2]}$ However, the expression patterns and pathogenic significance of the whole integrin family in SSc are still unclear.

Objectives: This study aimed at evaluating the integrin family gene expression in skin lesion from SSc patients and exploring its potential pathogenic mechanism. Methods: We utilized the public datasets of SSc skin tissue from Gene Expression Omnibus (GEO) database to analyze the expression and clinical significance of integrin family genes in SSc. In addition, functional enrichment and pathway analysis were also conducted.

Results: Compared with healthy controls, ITGA5, ITGA7, ITGA8, ITGB2, ITGB5, ITGAE and ITGB3BP were abnormally overexpressed in the skin of SSc. Further analysis indicated that ITGA5, ITGA7, ITGA8, ITGB2 and ITGB5 were positively correlated with modified Rodnan skin thickness score (mRSS), while ITGAE and ITGB3BP were negatively correlated with $\mathrm{mRSS}$ in SSc. Increased ITGB5 expression was associated with positive of anti-centromere antibody (ACA) Functional enrichment and pathway analysis showed that integrin members had multiple functions in SSc. Among them, ITGA5, ITGB2 and ITGB5 might synergistically promote SSc through affecting extracellular matrix (ECM) turn over, ECM-receptor interaction, focal adhesion and leukocyte trans-endothelial migration. ITGA5 and ITGB5 also affected angiogenesis and endothelial cell function. In addition, ITGA5 was uniquely enriched for actin organization, ITGB5 was uniquely enriched for TGF- $\beta$ signaling, and ITGB2 was uniquely associated with immune cells activation.

Conclusion: Our results implied that integrins, especially ITGA5, ITGB5, ITGB2 participated in the process of inflammation, vasculopathy and fibrosis in SSc. Together, they might render important therapeutic targets for SSc.

\section{REFERENCES:}

[1] Brown M, O'Reilly S. The immunopathogenesis of fibrosis in systemic sclerosis. Clin Exp Immunol. 2019;195(3):310-321.

[2] Gerber, E.E., et al., Integrin-modulating therapy prevents fibrosis and autoimmunity in mouse models of scleroderma. Nature, 2013. 503(7474): p 126-30.

Disclosure of Interests: None declared

DOI: 10.1136/annrheumdis-2021-eular.3646

\section{Vasculitis - aetiology, pathogenesis and animal models}

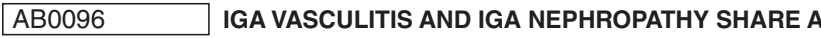 SIMILAR IL17A ASSOCIATION PATTERN}

D. Prieto-Peña ${ }^{1}$, F. Genre ${ }^{1}$, S. Remuzgo Martinez ${ }^{1}$, V. Pulito-Cueto ${ }^{1}$, B. AtienzaMateo $^{1,2}$, B. Sevilla ${ }^{3}$, J. Llorca ${ }^{4}$, N. Ortego ${ }^{5}$, L. Lera-Gómez ${ }^{1}$, M. Leonardo ${ }^{6}$,

A. Peñalba ${ }^{6}$, L. Martín-Penagos ${ }^{7}$, J. A. Miranda-Filloy ${ }^{8}$, J. Narváez ${ }^{9}$, L. Caminal Montero $^{10}$, P. Collado ${ }^{11}$, A. Fernandez-Nebro ${ }^{12}$, G. Díaz-Cordoves ${ }^{12}$,

S. Cigarrán ${ }^{13}$, J. Calviño ${ }^{14}$, C. Cobelo ${ }^{14}$, J. Sanchez Perez ${ }^{15}$, D. De Argila ${ }^{15}$,

E. Rubio-Romero ${ }^{16}$, M. Leon Luque ${ }^{16}$, J. M. Blanco-Madrigal ${ }^{17}$, E. Galindez ${ }^{17}$,

J. Martin Ibanez $^{18}$ S. Castañeda ${ }^{19}$ R. Blanco ${ }^{1}$, M. A. González-Gay ${ }^{1,20,21}$

R. López-Mejías ${ }^{1} .{ }^{1}$ Research Group on Genetic Epidemiology and 